\title{
Lifetimes of Magnons in Two-Dimensional Diluted Ferromagnetic Systems
}

\author{
Akash Chakraborty, Paul Wenk, and John Schliemann \\ Institut für Theoretische Physik, Univeristät Regensburg, 93040 Regensburg, Germany
}

(Dated: August 19, 2021)

\begin{abstract}
Spin dynamics in low dimensional magnetic systems has been of fundamental importance for a long time and has currently received an impetus owing to the emerging field of nanoelectronics. Knowledge of the spin wave lifetimes, in particular, can be favorable for future potential applications. We investigate the low-temperature spin wave excitations in two-dimensional disordered ferromagnetic systems, with a particular focus on the long wavelength magnon lifetimes. A semianalytical Green's functions based approach is used to determine the dynamical spectral functions, for different magnetic impurity concentrations, from which the intrinsic linewidth is extracted. We obtain an unambiguous $q^{4}$ scaling of the magnon linewidth which is ascribed to the disorder induced damping of the spin waves, thereby settling a longstanding unresolved issue on the wave-vector dependence. Our findings are also in good agreement with previous theoretical studies on Heisenberg ferromagnets. Additionally, we demonstrate the futility of using the low moments associated with the spectral densities to evaluate the magnon dispersions and lifetimes.

PACS numbers: 75.30.Ds, 73.21.-b, 75.50.Pp
\end{abstract}

\section{INTRODUCTION}

Two-dimensional magnetic systems have been a subject of intensive investigation for almost half a century now. Both ferro- and anti-ferromagnetic systems have been studied extensively, experimentally as well as theoretically, revealing a myriad of interesting properties including the discovery of high-temperature superconductivity in doped two-dimensional cuprates ${ }^{1}$. One of the important aspects of these systems which has continued to attract interest is the magnetic excitations which are of fundamental relevance to understand the spin dynamics. Knowledge of the collective spin wave excitations can provide valuable insight into their dynamical response as well as their thermodynamic behavior. The advancement of experimental techniques, such as ferromagnetic resonance spectroscopy and inelastic neutron scattering, have been of immense help in exploring this field meticulously ${ }^{2,3}$. Inelastic neutron scattering is one of the most powerful and versatile tools as the long wavelength spin excitations, better known as magnons, can be probed directly and accurately. One of the most thoroughly studied systems, in this context, is the two-dimensional Heisenberg anti-ferromagnet $\mathrm{Rb}_{2} \mathrm{Mn}_{1-x} \mathrm{Mg}_{x} \mathrm{~F}_{4}{ }^{4-6}$, which was investigated by means of neutron scattering techniques to study the low-temperature magnetic excitations, viz. the magnon dispersion, linewidths, and lineshapes, as well as the critical exponents near the transition temperature. This led to similar studies on $\mathrm{K}_{2} \mathrm{Cu}_{1-x} \mathrm{Zn}_{x} \mathrm{~F}_{4}{ }^{7}$, which is a quasi bi-dimensional ferromagnet. In the aforementioned studies, good agreement with numerical calculations, available at that time, was also reported. However, despite the existence of innumerable studies, one important feature which has eluded understanding, over the decades, is the wave-vector dependence of the magnon lifetime (inversely proportional to the linewidth), especially in the long wavelength limit $(q \rightarrow 0)$.
Spin waves in Heisenberg ferromagnets, in the lowenergy limit, were studied theoretically as early as the sixties by Murray ${ }^{8}$. The author calculated the spin wave energies and the scattering cross section, within the Born approximation, and reported a $q^{5}$ scaling of the magnon lifetime. The exchange interactions, in this case, were restricted to nearest neighbors only. Similar $q^{5}$ dependence was also found in amorphous Heisenberg ferromagnets, in the low-temperature and long wavelength limit, by using an effective medium approximation ${ }^{9}$. In this case, however, spatially dependent extended couplings were assumed between the magnetic sites. Based on Green's functions calculations, Mano $^{10}$ also predicted an identical behavior of the lifetime in the long wavelength limit. The finite linewidth of the excitations, which increased rapidly with decreasing wavelength, was attributed to the randomness in the magnitude of the spins. Also the discrepancy between the observed magnetization behavior and that predicted by elementary spin wave theory was believed to originate from this finite linewidth of the spin waves. On the contrary, similar spin wave studies in amorphous ferromagnets by Kaneyoshi ${ }^{11}$ led to a $q^{7}$ dependence of the linewidth. This was an outcome of using a quasi-crystalline approximation, which is essentially a virtual-crystal-like approach. Within this approximation, the magnon dispersion reduces to that of an ideal crystal, wherein the disorder effects are completely neglected. In yet another study, based on the two-magnon interaction theory of Heisenberg ferromagnets, a leading order $q^{2}$ scaling of the magnon lifetime was suggested by Ishikawa et al. ${ }^{12}$. However, in most of the aforesaid studies, the systems under consideration were threedimensional Heisenberg ferromagnets and there was no clear mention of the dimensional dependence. It was only later that Christou and Stinchcombe ${ }^{13}$ investigated the low-temperature spin excitations in bond-diluted Heisenberg ferromagnets from a more generalized perspective. Using a diagrammatic perturbation theory, the authors 
obtained a $q^{d+2}(d>1$, is the dimensionality) scaling of the magnon linewidth. Although the discussion was extended to the more relevant site-diluted systems, the exchange interactions were again restricted to nearest neighbors only.

Thus, the lack of a general accord on the issue of linewidth scaling becomes apparent from the widely varying predictions available in the literature. Moreover, considerable attention and interest have also been devoted to the case of anti-ferromagnets, including even lately ${ }^{14}$. In a very recent study ${ }^{15}$, on three-dimensional disordered ferromagnets, a $q^{5}$ scaling of the magnon linewidth, in the long wavelength limit, was reported using similar numerical approaches as implemented here. This served as a further motivation behind the current study of the magnetic excitations in two-dimensional ferromagnetic systems, with a view to identify the dimensional dependence of the scaling of the magnon lifetimes. Also a proper knowledge of the lifetimes is not only of fundamental interest but can also be of practical importance, as we shall discuss later. In this article, we provide a comprehensive and detailed analysis of the low-temperature spin wave excitations in two-dimensional site-diluted ferromagnets, in the presence of extended exchange interactions. The calculations have been performed on sufficiently large system sizes and a proper statistical sampling over disorder is also taken into account. We lay special emphasis on the correct evaluation of the magnon linewidths in the long wavelength limit. In the process, we demonstrate that determining the correct wave-vector dependence of the lifetimes constitutes a non-trivial task. In addition, we also discuss the nature of the magnon density of states, the spectral functions, as well as the magnon dispersion over a relatively broad concentration range.

\section{HEISENBERG MODEL AND EXCHANGE COUPLINGS}

We start with the Hamiltonian describing $N_{i m p}$ interacting spins $\left(\mathbf{S}_{i}\right)$ randomly distributed on a square lattice of $N$ sites, given by the dilute Heisenberg model

$$
H=-\sum_{i, j} J_{i j} p_{i} p_{j} \mathbf{S}_{i} \cdot \mathbf{S}_{j}
$$

where the sum $i, j$ runs over all sites and the random variable $p_{i}=1$ if the site is occupied by an impurity or otherwise zero. We consider classical spins $\left(\left|\mathbf{S}_{i}\right|=S\right)$ on a square lattice, with lattice spacing $a$, and with periodic boundary conditions. The distribution of the spins, in this case, is completely random and uncorrelated; in other words the probability of a spin to be placed at site $i$ is independent of the neighboring sites. This is in contrast to a previous study ${ }^{16}$ on the magnetic excitations in inhomogeneous diluted systems, where well-defined spherical clusters of spins were considered. Spin-orbit coupling is neglected as this would lead to anisotropy in the system which is not the primary focus here. The effects of spin-orbit on the magnon lifetimes in two-dimensional systems were studied in ${ }^{17}$. All calculations, in the present work, are performed at $T=0 \mathrm{~K}$. The concentration of magnetic impurities in the system is denoted by $x\left(=N_{i m p} / N\right)$. The Hamiltonian, Eq. (1), is treated within the self-consistent local random phase approximation (SC-LRPA), which is essentially a semi-analytical approach based on (finite temperature) Green's functions. Within this approach, the retarded Green's functions are defined as

$$
G_{i j}^{c}(\omega)=\int_{-\infty}^{\infty} G_{i j}^{c}(t) e^{i \omega t} d t
$$

where $G_{i j}^{c}(t)=-i \theta(t)\left\langle\left[\mathbf{S}_{i}^{+}(t), \mathbf{S}_{j}^{-}(0)\right]\right\rangle$, describe the transverse spin fluctuations, and $\langle\ldots\rangle$ denotes the expectation value, and ' $c$ ' the disorder configuration index. After performing the Tyablikov decoupling ${ }^{18-20}$ (assuming magnetization along the $z$-axis) of the higher-order Green's functions which appear in the equation of motion of $G_{i j}^{c}(\omega)$, we obtain

$$
\left(\omega \mathbf{I}-\mathbf{H}_{e f f}^{c}\right) \mathbf{G}^{c}=\mathbf{D}
$$

where $\mathbf{H}_{e f f}^{c}, \mathbf{G}^{c}$, and $\mathbf{D}$ are $N_{i m p} \times N_{i m p}$ matrices. The effective Hamiltonian matrix elements are

$$
\left(\mathbf{H}_{e f f}^{c}\right)_{i j}=-\left\langle S_{i}^{z}\right\rangle J_{i j}+\delta_{i j} \sum_{l}\left\langle S_{l}^{z}\right\rangle J_{l j}
$$

and the diagonal matrix

$$
D_{i j}=2\left\langle S_{i}^{z}\right\rangle \delta_{i j} .
$$

For a given temperature and disorder configuration, the local magnetizations $\left\langle S_{i}^{z}\right\rangle\left(i=1,2, \ldots, N_{i m p}\right)$ have to be calculated self-consistently. However, since we are interested at $T=0 \mathrm{~K}$, where the ground state is assumed to be fully polarized, all $\left\langle S_{i}^{z}\right\rangle$ are equal to $S$ in this case. We shall not go into further details of the method here, as the accuracy and reliability of the SC-LRPA to handle disorder (dilution) effects in different contexts have been discussed and established on numerous previous occasions (for details see Refs. 21-23). The virtual crystal approximation, as a possible alternative approach, fails in these systems as will be discussed in Sec. V.

The exchange interactions are assumed to be of the form $J_{i j}=J_{0} r_{i j}^{-\alpha}$, where $r_{i j}=\left|\mathbf{r}_{i}-\mathbf{r}_{j}\right|$. In most of the previous studies the exchange couplings were restricted to nearest neighbors only, but in realistic systems these interactions extend well beyond the nearest neighbors. Moreover, the choice of the couplings is motivated by the theoretical proposition put forward in Ref. 24, wherein the author extends the Mermin-Wagner theorem ${ }^{25}$ to Heisenberg and $X Y$ systems with long-range interactions. It is stated that a $d$-dimensional system $(d=1$ or 2 ) with monotonically decaying interactions as $\left|J_{\mathbf{r}}\right| \propto r^{-\alpha}$ cannot have ferro- or anti-ferromagnetic long-range order at $T>0$, if $\alpha \geq 2 d$. For RKKY-like interactions 
(long-range oscillatory nature) magnetic order could be strictly ruled out for the one-dimensional systems, but only for certain cases in the two-dimensional ones. It was later proved by Loss, Pedrocchi, and Leggett ${ }^{26}$, again as an extension of the Mermin-Wagner theorem, that no long-range magnetic order is possible in one or twodimensional systems at a finite temperature, in the presence of RKKY interactions. The choice of exponentially decaying couplings can also be ruled out in this case as they satisfy the Mermin-Wagner theorem trivially. Hence, this led us to the choice of the exponent $\alpha=3$ for the couplings, which implies that in our two-dimensional systems long-range (ferro-)magnetic order at a finite temperature is not excluded by the Mermin-Wagner theorem. Also, since the couplings are isotropic and all ferromagnetic $\left(J_{i j}>0\right)$ there is no frustration expected and hence the collinear state can be safely assumed to be the ground state. This in turn leads to only positive eigenvalues in the magnon spectrum which will become clear in the following calculations of the magnon DOS.

\section{MAGNON DENSITY OF STATES AND SPECTRAL FUNCTION}

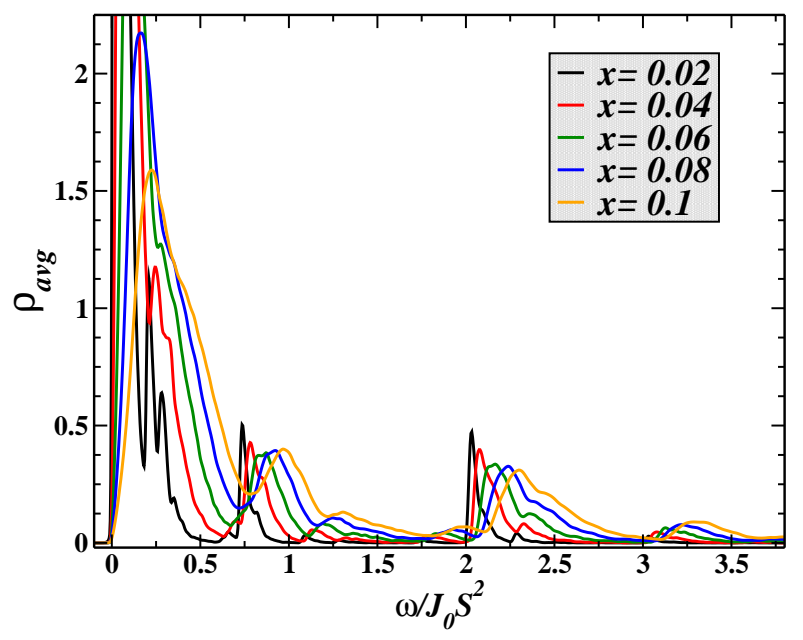

FIG. 1. (Color online) Average magnon DOS $\rho_{\text {avg }}$ as a function of energy $\omega$ plotted for different concentrations $x$.

From the retarded Green's functions defined above one can calculate the average magnon density of states (DOS), which is given by $\rho_{\mathrm{avg}}(\omega)=\left(1 / N_{\mathrm{imp}}\right) \sum_{i} \rho_{i}(\omega)$, where $\rho_{i}(\omega)=-1 /(2 \pi S) \Im\left[G_{i i}(\omega)\right]$ is the local magnon DOS. Fig. 1 shows the average magnon DOS as a function of the energy for different impurity concentrations. The DOS have been averaged over a hundred disorder configurations, although it was found that typically 25 configurations were sufficient for each impurity concentration. We observe irregular features in the DOS which become more pronounced with increase in dilution. On decreasing the concentration from $x=0.1$ to $x=0.02$, a significant increase in weight around the low energy end of the spectrum is observed. This increase in weight is attributed to the increase in the fraction of impurities which are weakly connected to the rest. These isolated impurity regions have their own zero-energy modes which in turn contribute to the DOS at the low energies. In order to gain a better insight into this behavior we look at the distribution of the local DOS shown in Fig. 2(a) and 2(b), at two different energies $2.2 J_{0} S^{2}$ and $3.2 J_{0} S^{2}$, respectively for $x=0.1$. Here we can clearly identify certain impurity regions, of typically two or three impurities, which are weakly coupled to the surrounding impurities. These can be seen to make a higher contribution to the DOS. (For more details see Fig. 7, App.). Note that the distribution shown corresponds only to a part of the lattice from a $200 a \times 200 a$ system. With increasing dilution the average separation between the spins increases and hence the effective coupling decreases. This accounts for the increase in the irregular features observed in the DOS for $x=0.02$.

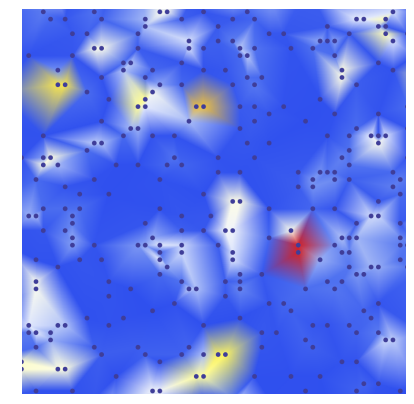

(a)

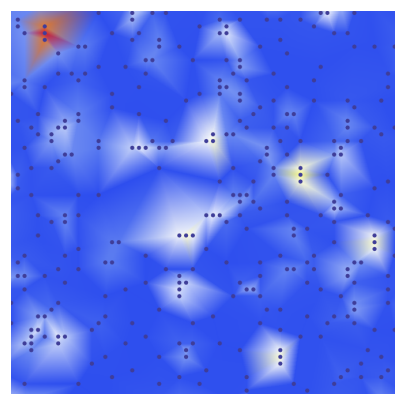

(b)

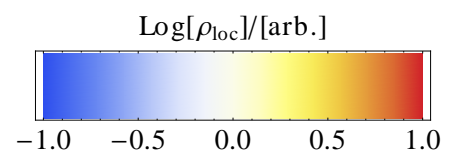

FIG. 2. (Color online) Distribution of the local magnon DOS at energies (a) $\omega /\left(J_{0} S^{2}\right)=2.2$, and (b) $\omega /\left(J_{0} S^{2}\right)=3.2$, for an impurity concentration of $x=0.1$. Shown is a part of the lattice of size $L=200 a$ in coordinate space. The dots indicate the positions of the spins $\mathbf{S}_{i}$.

The dynamical spectral function, also known as the structure factor, provides valuable insight into the underlying spin dynamics of a system. Experimentally this can be probed by inelastic neutron scattering and ferromagnetic resonance to a good accuracy. The averaged spectral function is defined by

$$
A(\mathbf{q}, \omega):=-\left\langle\frac{1}{2 \pi S} \Im\left[G^{c}(\mathbf{q}, \omega)\right]\right\rangle_{c},
$$

where $G^{c}(\mathbf{q}, \omega)$ is the Fourier transform of the retarded Green's function $G_{i j}^{c}(t)$, and $\langle\ldots\rangle_{c}$ denotes the configuration average. Fig. 3 shows the averaged spectral functions as a function of energy for four different concentrations. The $A(\mathbf{q}, \omega)$ 's are averaged over a few hundred 

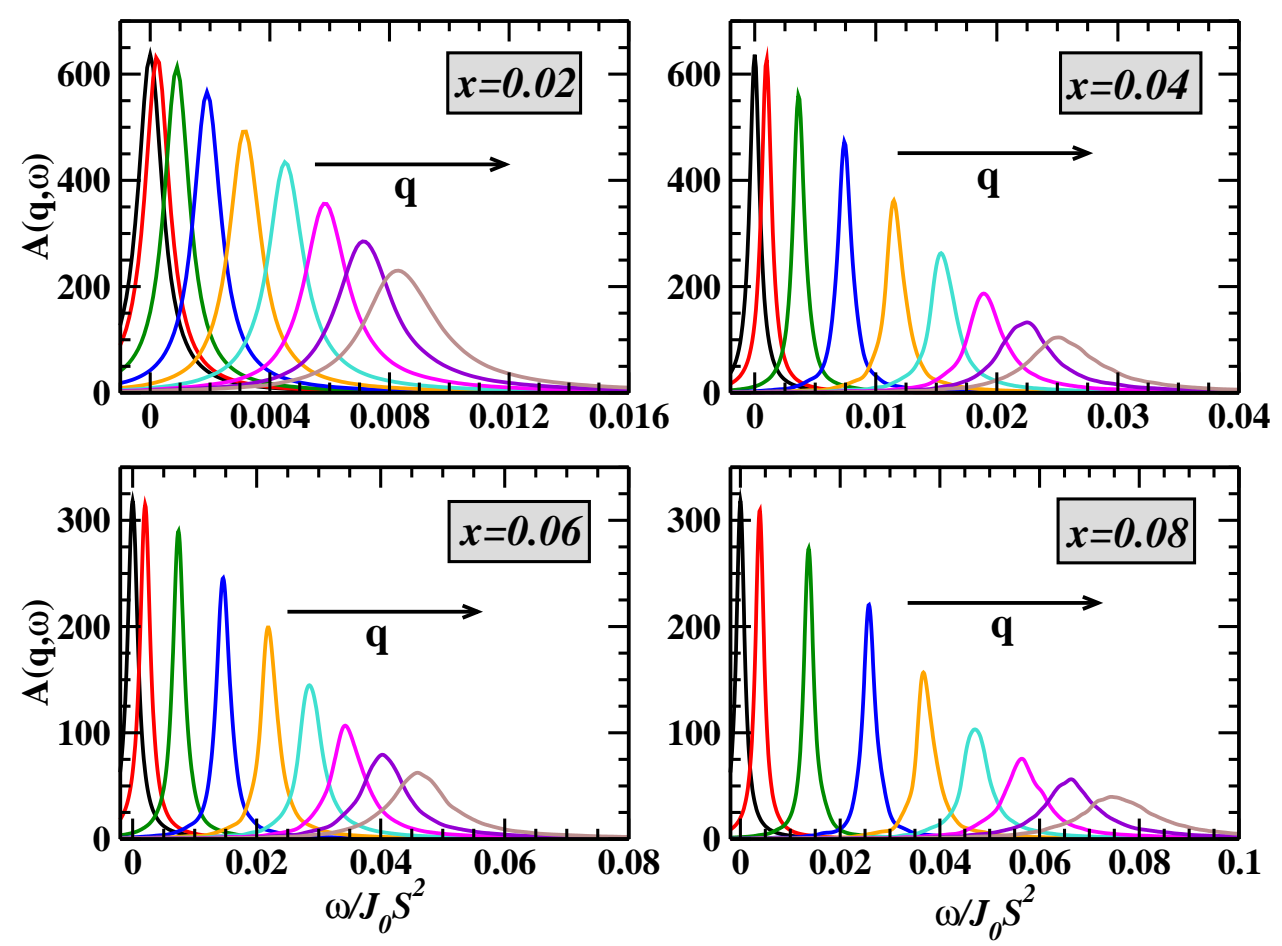

FIG. 3. (Color online) Average spectral function $A(\mathbf{q}, \omega)$ as a function of the energy with $\mathbf{q}=n(2 \pi / L a)\{1,0\}^{\top}$ (where $n \in \mathbb{N}$ ), corresponding to four different concentrations $x$. The system size is $L^{2}=300 a \times 300 a$.

disorder configurations, and the results are plotted only in the $\left[\begin{array}{ll}1 & 0\end{array}\right]$ direction of the Brillouin zone, for progressively increasing momentum $\mathbf{q}$, since the focus is on the long wavelength regime here. It should be noted that the $\left[\begin{array}{ll}0 & 1\end{array}\right]$ direction is equivalent to the $\left[\begin{array}{ll}1 & 0\end{array}\right]$ direction in this case, due to the lattice symmetry. Also note that for $q \gg 2 \pi /(L a)$ the deviation from rotation invariance is not negligible. Well-defined excitations are found to exist only for small values of $\mathbf{q}$, in each case. For increasing $\mathbf{q}$, the excitation peaks become broader and develop a tail extending toward the higher energies. On decreasing the concentration from 0.08 to 0.02 the zone of stability of the well-defined magnon modes is found to decrease by almost one order of magnitude. Also the excitations become increasingly asymmetric with increase in the momentum. This increase in asymmetry is associated with the crossover from propagating low-energy spin waves to localized or quasi-localized excitations (fractons) ${ }^{27}$ at higher energies. Here, the term localized implies that the excitations are quite broad in energy at fixed wavevectors, or rather quantitatively the excitation energy is much larger than the linewidth (i.e. the full-width at halfmaximum).

The nature of the spectral functions is similar to what was observed by neutron scattering experiments in $\mathrm{Mn}_{x} \mathrm{Zn}_{1-x} \mathrm{~F}_{2}{ }^{28,29}$, which is a three-dimensional randomly diluted anti-ferromagnet. The authors measured sharp spin waves near the zone center which broadened progressively with the wave vector approaching the zoneboundary. These findings were attributed to a crossover from low-energy extended spin waves (magnons) to localized high-energy excitations (fractons), which was further consistent with the theoretical conjecture of fractons in disordered percolating networks ${ }^{27,30}$. A recent numerical study ${ }^{31}$ on site-diluted two-dimensional antiferromagnets also reveal the existence of localized excitations at high energies. The authors evaluated the inverse participation ratio (IPR), for different dilutions and different system sizes in order to establish the nature (extended or localized) of the states, although the largest system size studied was only $32 a \times 32 a$. These studies provide relevance and also additional motivation to study the two-dimensional ferromagnets from this aspect. The proper and accurate evaluation of the spectral functions, as we shall see in what follows, constitutes a vital task since the magnon dispersion as well as the lifetime can be directly extracted from them.

\section{MOMENTS ANALYSIS}

Before embarking into further details of the long wavelength magnon properties, we define the moments associated with the spectral density. The $n$-th moment is 


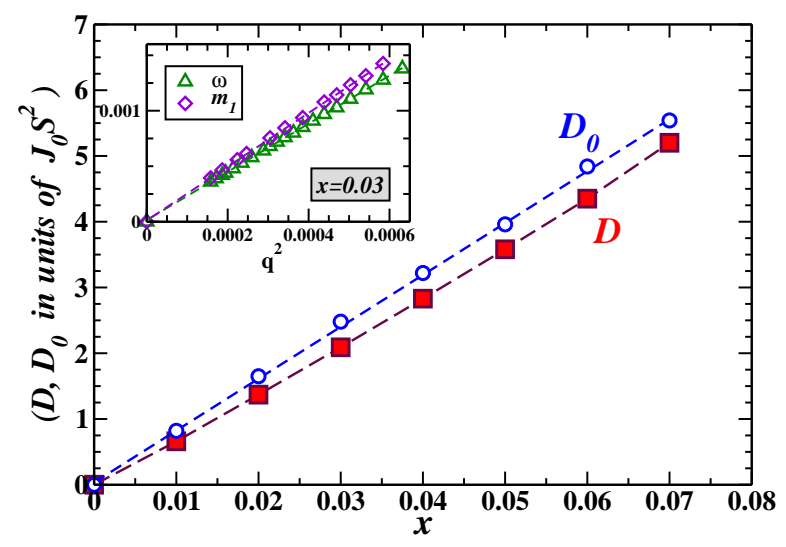

FIG. 4. (Color online) Spin stiffness $D$ and effective spin stiffness $D_{0}$ as a function of $x . \quad\left(m_{1}(q) \approx D_{0} q^{2}\right.$, where $m_{1}$ is the first moment associated to the spectral density). The inset shows a comparison of the excitation energies extracted from $A(\mathbf{q}, \omega)$ and the first moments $m_{1}$, respectively, for the case of $x=0.03$.

defined by

$$
m_{n}(\mathbf{q})=\int_{0}^{\infty} \omega^{n} A(\mathbf{q}, \omega) d \omega
$$

In the limit $q \rightarrow 0$, it can be shown that $m_{1}(\mathbf{q}) \approx D_{0} q^{232}$, where we call $D_{0}$ as the effective spin wave stiffness. It is also well known that in the long wavelength limit the dispersion in ferromagnetic systems is quadratic in $q, \omega(\mathbf{q}) \approx D q^{2}$, where $D$ denotes the spin stiffness coefficient. The moments, as sometimes found in the literature ${ }^{33,34}$, are used in the spectral function analyses as a good approximation to estimate the excitation energy and linewidth, especially in the presence of disorder. Nonetheless, the accuracy and the viability of this assumption is subject to further examination. In order to address this, as a first step, we numerically calculated the dispersion from the first moment and then compared it to the real excitation energy $\omega(\mathbf{q})$ extracted from the $A(\mathbf{q}, \omega)$ peaks shown in Fig. 3 . The results for the particular case of $x=0.03$ are plotted in the inset of Fig. 4. As can be seen, in the small $q$ limit, both $m_{1}(q)$ and $\omega(\mathbf{q})$ are linear in $q^{2}$ but the first moment fairly overestimates the real magnon energies. This is better reflected when we extract the respective spin stiffness coefficients, $D_{0}$ from $m_{1}(q)$ and $D$ from $\omega(\mathbf{q})$, and plot them against the concentration as shown in Fig. 4 . For all considered $x$, the effective spin stiffness is larger than the actual spin stiffness, overestimating by $15-20 \%$ in each case. This clearly demonstrates that the first moment is not a reliable quantity to evaluate the spin stiffness in these diluted systems as it fails to reproduce the magnon energies precisely.

The other relevant quantity of interest is the intrinsic linewidth of the magnetic excitations. The linewidth

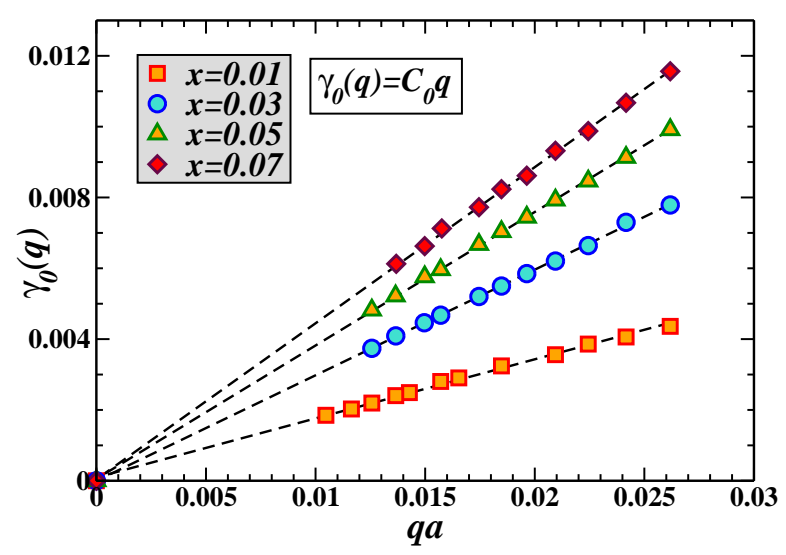

FIG. 5. (Color online) Effective linewidth $\gamma_{0}$ (in units of $J_{0} S^{2}$ ) as a function of $q$ (in the [1 0 ] direction), for different concentrations $x$, Eq. (8). The dashed lines indicate the linear fits.

gives a measure of the excitations' broadening due to disorder, which maybe magnetic or structural disorder, or due to the magnon-magnon interactions. One can obtain the linewidth from the second moment from the relation ${ }^{33}$

$$
\gamma_{0}(\mathbf{q})=\sqrt{m_{2}(\mathbf{q})-m_{1}^{2}(\mathbf{q})}
$$

where $\gamma_{0}(\mathbf{q})$ is the effective linewidth. In Fig. 5 we have plotted this effective linewidth as a function of $\mathbf{q}$ for four different impurity concentrations. We find that in the small- $q$ limit the linewidth is linear in $q$ for all considered $x$. The same holds true for all other intermediate concentrations, which are not shown here. Consequently, we end up with $\omega \propto q^{2}$ and $\gamma_{0} \propto q$, in the limit $q \rightarrow 0$. This indicates that the magnetic excitations are incoherent or localized around the $\Gamma$-point, since $\gamma_{0}>\omega$. However, this is somehow contrary to what we have observed in the spectral functions shown in Fig. 3, where the excitations are well defined for small $q$ values. Hence, we can safely assume that the effective linewidth obtained from the moments does not correspond to the real linewidth of the excitations. The same discrepancy was also demonstrated for the case of $\mathrm{Ga}_{1-x} \mathrm{Mn}_{x} \mathrm{As}^{32}$, a wellknown III-V diluted ferromagnetic semiconductor, where the lattice has an fcc structure. Note that similar linear $q$-dependence, obtained from the moments analysis, was reported by the authors in disordered double-exchange systems ${ }^{34}$. Determining the correct $q$-dependence of the intrinsic linewidth, in the long wavelength limit, requires further detailed analysis which is elucidated in the following. 


\section{SCALING OF MAGNON LIFETIME}

We extract the linewidth, which is the full-width at half-maximum, directly from the magnon spectral functions (Fig. 3) corresponding to the first non-zero $q$ values from different system sizes. The extracted linewidths are plotted as a function of the wave-vector in Figs. 6(a) and $6(\mathrm{~b})$, for $x=0.03$ and 0.05 , respectively. In order to have sufficiently small $q$ values, and also check for the probable finite-size effects we have performed the calculations on system sizes ranging from $200 a \times 200 a$ up to $500 a \times 500 a$. The linewidth data are averaged over one hundred disorder configurations and the error bars corresponding to the standard deviation are contained within the symbols. Now, since we are interested in the $q \rightarrow 0$ regime, we focus on a restricted region of the $q$ values, (highlighted by the shaded regions in the plots), in order to give more weight to the smallest available $q$ 's. We remark that the limit considered for the shaded regions only serve as an approximate value and not as a clear demarcation of the $q$ regime, defining the long wavelength limit. Note that the value of $\ln (q a) \approx-4$ corresponds to a value $q a \approx 0.02$. To determine the $q$-dependence we use a linear fit of the form $n \ln (q a)+C$, (with $n=3$, 4 , and 5) for the data within these shaded regions. As can be clearly seen for both cases, $x=0.03$ and 0.05 , it is the $n=4$ fit (denoted by the solid line) which best describes the linewidth behavior in this region. Beyond this region, the linewidth begins to deviate from this behavior although the deviations are less for $x=0.05$ compared to $x=0.03$. Also note that the same $q$-scaling was observed for the other concentrations as well. This clearly shows that in the long wavelength limit and at low temperatures the intrinsic linewidth actually scales as $q^{4}$ in these two-dimensional systems. Our findings are interestingly in good agreement with the prediction of a $q^{d+2}$ - dependence reported in Ref. 13. This agreement is not obvious since in the latter work a different analytical approach, based on diagrammatic perturbation theory, was used and also the couplings were restricted to nearest neighbors only. Whereas our study is more general in the sense that the couplings are extended, as well as the linewidth is extracted directly from the magnon spectral functions. In this context, it is worth mentioning that studies based on virtual-crystal-like approaches often lead to an infinite lifetime, since the spin fluctuations are unaccounted and the disorder effects are neglected, implying no mechanism for magnon decay. However, disorder plays an essential role, as shown here, in leading to a finite lifetime in these systems. From this $q^{4}$ scaling we infer that, in the long wavelength limit, the linewidth is actually smaller than the excitation energy which was qualitatively clear from the well-defined peaks observed around the $\Gamma$-point in the spectral functions. Nevertheless, as we have seen, it is difficult to identify precisely the values of $q$ below which this behavior holds and these values, in turn, should also depend on the concentration $x$. Similar difficulties were also demonstrated in the case of three-dimensional systems ${ }^{15}$ where a $q^{3}$ behavior, instead of $q^{5}$, was observed if the considered $q$ values were not sufficiently small. Apparently, in the present case we do not observe any clear crossover from the $q^{4}$ scaling to any other form within the considered range of the wave-vectors. We also conclude that the scaling of the linewidths does in fact depend on the dimensionality.
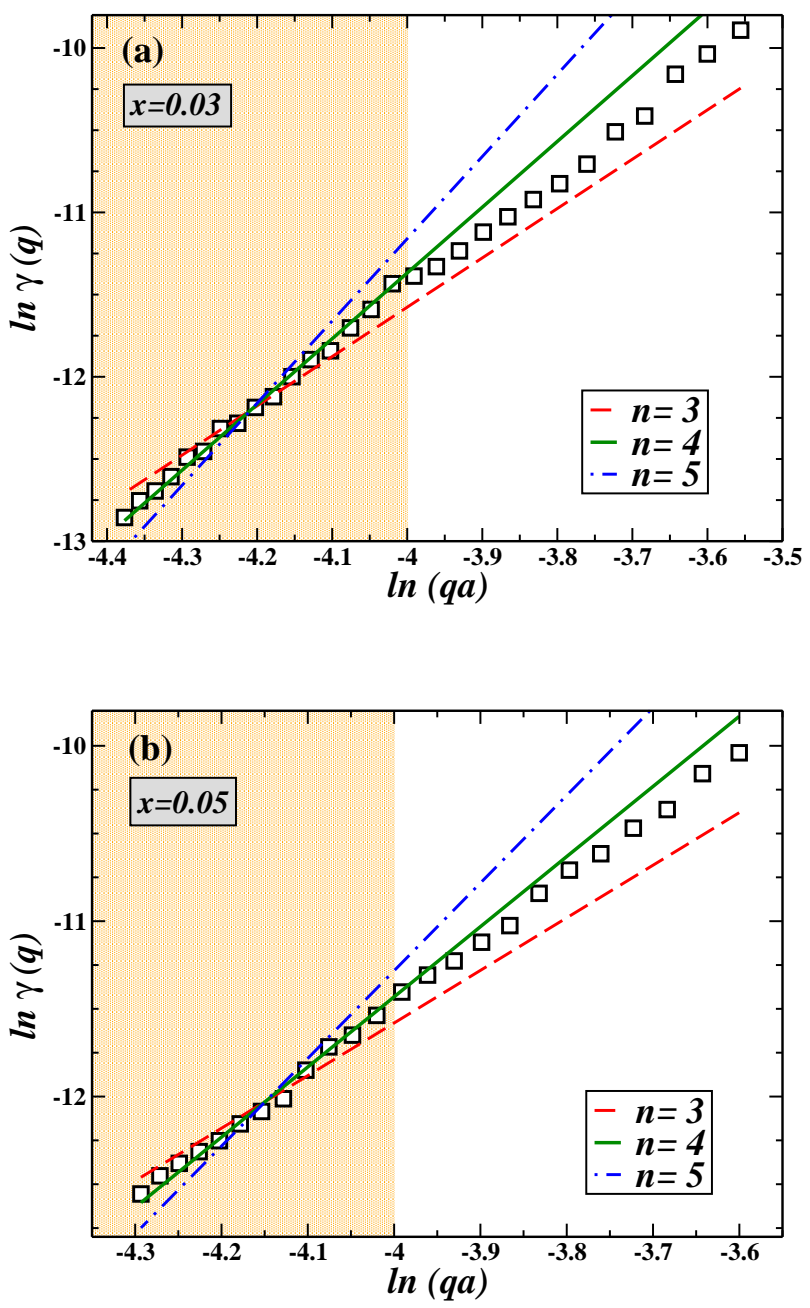

FIG. 6. (Color online) Logarithm of the magnon linewidth $\gamma$ (in units of $J_{0} S^{2}$ ) as a function of $\ln (q a)$, for (a) $x=0.03$, and (b) $x=0.05$. Dashed (red), solid (green), and dot-dashed (blue) lines indicate linear fits of the form $n \ln (q a)+C,(n=3$, 4 , and 5 ), for the linewidth data within the shaded region.

As already mentioned, the energy and the linewidth calculated from the moments do not coincide with the real ones extracted from the spectral function. The reason behind is that moments can only reproduce the characteristic features of a distribution when they are perfectly symmetric, such as a Gaussian or a Lorentzian. In the present case, the spectral functions are actually asymmetric and hence the moments prove to be inappropriate to estimate the real line shape and the peak positions. In 
the clean case (absence of any disorder) one is likely to get reliable results from the moments analysis of the spectral functions as the excitations can be completely symmetric. However, disorder leads to a strong asymmetry of the excitations, as we have seen in the present case. There is a considerable broadening in the spectrum observed especially close to the zone boundary. Thermal fluctuations also play an important role in these systems, but since we focus only on the low-temperature excitations we can neglect the thermal effects here. Further experimental studies to quantitatively examine the linewidth in these compounds could prove to be very useful.

\section{CONCLUSION}

We have addressed the low temperature spin excitations in two-dimensional diluted Heisenberg systems, with a particular focus on the long wavelength limit. A self-consistent Green's functions based approach is used to evaluate the magnon DOS and the dynamical spectral functions. Well-defined excitations are observed only in a restricted region of the Brillouin zone, around the $\Gamma$ point. It is demonstrated that determining the correct wave-vector dependence of the magnon linewidth in diluted systems is not an ordinary task. Contrary to some previous studies, we have shown that the moments associated with the spectral function are inappropriate to determine the linewidth or the excitation energies. The moments overestimate the real spin stiffness as well as provide a linear $q$-dependence of the linewidth, implying incoherent excitations in the limit $q \rightarrow 0$. However, this is found to be inconsistent with the stiffness and the linewidth extracted from the calculated spectral functions. In the long wavelength limit, the linewidth in fact scales as $q^{4}$ in two-dimensional systems, for a wide range of impurity concentrations. The discrepancy arises due to the inability of the moments to reproduce the asymmetry in the excitation peaks. The origin of this asymmetry, and thus a finite lifetime, is ascribed to the disorder induced broadening of the spin waves. Hence, this underlines the importance of the disorder effects in these systems and we emphasize that the failure to properly account for them will certainly result in an incorrect wave vector dependence of the linewidth.

Most data storage devices, in nowadays spintronics, try to manipulate the dynamical motion of spins. From this perspective, a precise knowledge of the excitations' lifetime (inversely proportional to the linewidth) could be of practical relevance. For example, a short lifetime is important for memory devices to leave a bit in a steady state after a read-in or read-out operation. On the contrary, a longer lifetime is advantageous for the unhampered transmission of signals in inter-chip communications. It would be equally interesting to look into the temperature effects on the spin dynamics, in particular the linewidth, where in addition to disorder the thermal effects also play a vital role. However, this is beyond the scope of the current work. The present findings provide qualitative insights into the low temperature excitations and the magnon lifetimes in two-dimensional ferromagnets, and could serve as a firm basis for future research on complex disordered magnets. More experimental studies oriented in this direction are also highly desirable to resolve the controversy arising from the numerous theoretical proposals.

\section{ACKNOWLEDGMENTS}

We acknowledge financial support by DFG within the collaborative research center SFB 689. AC would like to thank Georges Bouzerar for insightful comments and discussions.

\section{Appendix A: Impurity Configuration Energies}

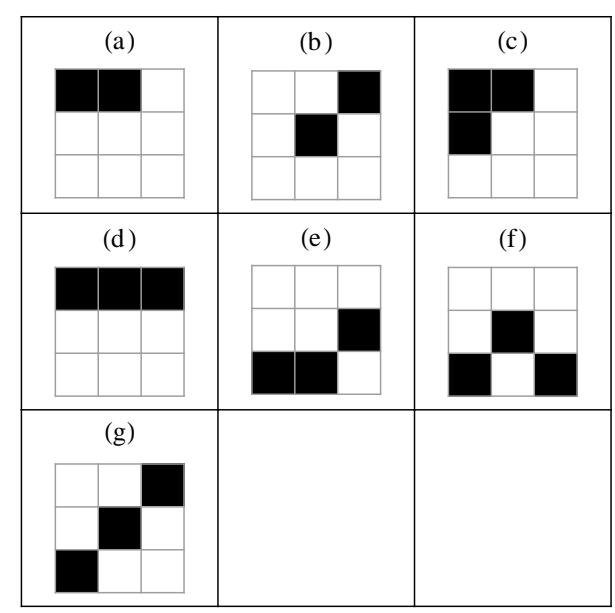

FIG. 7. Basic impurity configurations on a square lattice which give a high contribution to the magnon DOS.

The peaks in the averaged magnon DOS in the diluted case, Fig. 1, can be related to different configurations of some few impurities in coordinate space on the square lattice. This can be motivated by considering the distribution of the local magnon DOS in Fig. 2(a),2(b) which reveals clusters of less than four impurities up to an energy of $\omega /\left(J_{0} S^{2}\right) \approx 3$. Thus, the relevant small clusters which give rise to the magnon DOS peaks on a square lattice are identified to be those plotted in Fig. 7. The energies corresponding to the configurations (a)-(g) are given by

$$
\begin{aligned}
& E_{(\mathrm{a}) ; 1}=2 \\
& E_{(\mathrm{b}) ; 1}=2^{-\alpha / 2} E_{(\mathrm{a}) ; 1} \\
& E_{(\mathrm{c}) ; 1}=3 \\
& E_{(\mathrm{c}) ; 2}=1+2^{1-(\alpha / 2)} \\
& E_{(\mathrm{d}) ; 1}=3
\end{aligned}
$$




$$
\begin{aligned}
E_{(\mathrm{d}) ; 2} & =1+2^{1-\alpha} \\
E_{(\mathrm{e}) ; 1 / 2} & =1+2^{-\alpha / 2}+5^{-\alpha / 2} \pm 10^{-\alpha / 2}\left[2^{\alpha}\right. \\
-10^{\alpha / 2} & \left.\left(1+2^{\alpha / 2}\right)+5^{\alpha}\left(1-2^{\alpha / 2}+2^{\alpha}\right)\right]^{\frac{1}{2}} \\
E_{(\mathrm{f}) ; 1} & =2^{-\alpha / 2} E_{(\mathrm{c}) ; 1} \\
E_{(\mathrm{f}) ; 2} & =2^{-\alpha / 2} E_{(\mathrm{c}) ; 2} \\
E_{(\mathrm{g}) ; 1} & =2^{-\alpha / 2} E_{(\mathrm{d}) ; 1} \\
E_{(\mathrm{g}) ; 2} & =2^{-\alpha / 2} E_{(\mathrm{d}) ; 2}
\end{aligned}
$$

where the energy $E_{(.) ; p}$ is given in values of $J_{0} S^{2} / a^{\alpha}$, with $a$ being the lattice constant and the index $p$ the eigenvalue number (the ground-state energy zero has been excluded). The energies $E_{(.) ; p}$ are indicated in Fig. 8

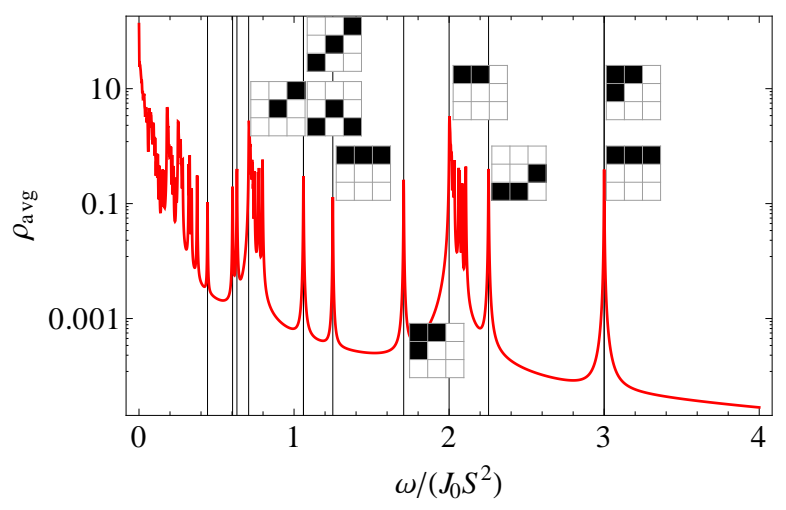

(a)

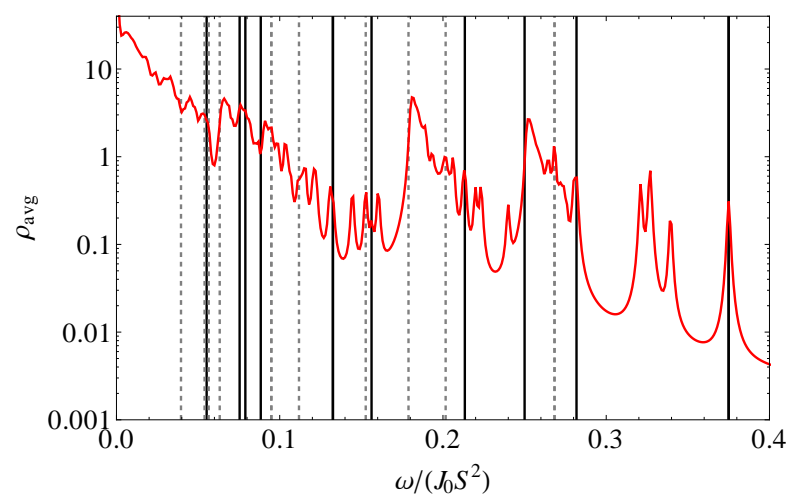

(b)

FIG. 8. (Color online) Averaged magnon DOS $\rho_{\text {avg }}$ of a small system $L=10 a$ with only three impurities.

(a) For specific maxima we show the corresponding impurity configuration in coordinate space on the square lattice. The energy corresponding to a configuration inset is on its LHS indicated by a solid line, according to Eq. A1- A11 with $\alpha=3$. (b) Zoom of plot (a) at small $\omega$ values. The solid lines indicate the energies after rescaling the lattice constant by two, the dashed lines a rescaling by $\sqrt{5}$.

by vertical lines. Fig. 8 shows the magnon DOS of a $10 a \times 10 a$ system with three impurities averaged over all possible configurations. A comparison of this results with the $x=0.02$ case in Fig. 1 (system size of $L=1340 a$ ) reveals indeed that the relevant energies $E \gtrsim 0.5 J_{0} S^{2}$ are given by the Eqs. (A1)-(A11), with $\alpha=3$. Many of the other configurations can be generated by a simple isotropic rescaling of the configurations shown in Fig. 7. The corresponding energies are indicated by dashed and solid lines in Fig. 8(b). To answer the question how spe-

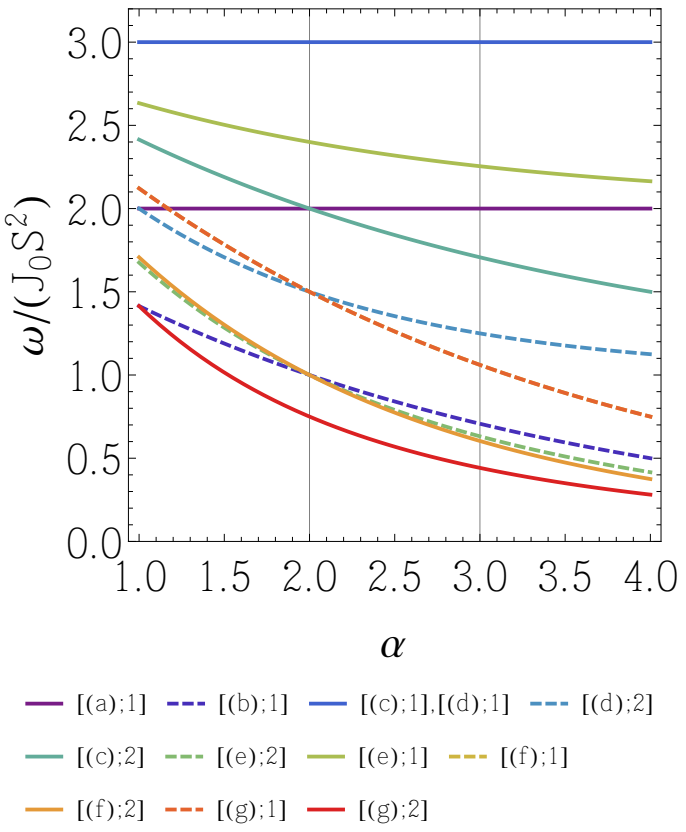

FIG. 9. (Color online) Magnon energies given by Eq. A1A11 for the corresponding impurity configurations plotted in Fig. 8 as function of the exponent $\alpha$ with $\left|J_{\mathbf{r}}\right| \propto r^{-\alpha}$.

cific the choice of the exponent $\alpha=3$ is, we plotted the energies Eq. A1- A11 of the magnon DOS peaks for different values of $\alpha$ in Fig. 9. We recall that a twodimensional system with monotonically decaying interactions cannot have ferro- or anti-ferromagnetic long-range order at $T>0$, if $\alpha \geq 4$. The plot shows that in the range of $2<\alpha<4$ the energies of the individual impurity configurations do not intersect each other. Thus, one can assume to have no significant and quantitative change in the physics if the exponent is varied within this range. 
1 J. G. Bednorz and K. A. Müller, Z. Phys. B 64, 189 (1986).

2 R. Coldea, S. M. Hayden, G. Aeppli, T. G. Perring, C. D. Frost, T. E. Mason, S.-W. Cheong, and Z. Fisk, Phys. Rev. Lett. 86, 5377 (2001).

3 M. D. Lumsden, A. D. Christianson, D. Parshall, M. B. Stone, S. E. Nagler, G. J. MacDougall, H. A. Mook, K. Lokshin, T. Egami, D. L. Abernathy, E. A. Goremychkin, R. Osborn, M. A. McGuire, A. S. Sefat, R. Jin, B. C. Sales, and D. Mandrus, Phys. Rev. Lett. 102, 107005 (2009).

4 R. A. Cowley, G. Shirane, R. J. Birgeneau, and H. J. Guggenheim, Phys. Rev. B 15, 4292 (1977).

${ }^{5}$ R. J. Birgeneau, J. Als-Nielsen, and G. Shirane, Phys. Rev. B 16, 280 (1977).

${ }^{6}$ R. J. Birgeneau, R. A. Cowley, G. Shirane, J. A. Tarvin, and H. J. Guggenheim, Phys. Rev. B 21, 317 (1980).

7 V. Wagner and U. Krey, Z. Phys. B 30, 367 (1978).

8 G. A. Murray, Proc. Phys. Soc. 89, 87 (1966).

${ }^{9}$ V. A. Singh and L. M. Roth, J. Appl. Phys. 49, 1642 (1978).

10 H. Mano, J. Phys. Soc. Jpn. 51, 3157 (1982).

11 T. Kaneyoshi, J. Phys. Soc. Jpn. 45, 1835 (1978).

12 Y. Ishikawa, K. Yamada, K. Tajima, and K. Fukamichi, J. Phys. Soc. Jpn. 50, 1958 (1981).

13 A. Christou and R. B. Stinchcombe, J. Phys. C 19, 5895 (1986).

14 S. P. Bayrakci, D. A. Tennant, Ph. Leininger, T. Keller, M. C. R. Gibson, S. D. Wilson, R. J. Birgeneau, and B. Keimer, Phys. Rev. Lett. 111, 017204 (2013).

15 A. Chakraborty and G. Bouzerar, J. Magn. Magn. Mater. 381, 50 (2015).

16 A. Chakraborty, P. Wenk, S. Kettemann, R. Bouzerar, and G. Bouzerar, New. J. Phys. 16, 033004 (2014).

17 Kh. Zakeri, Y. Zhang, T.-H. Chuang, and J. Kirschner, Phys. Rev. Lett. 108, 197205 (2012).
18 R. A. Tahir-Kheli and D. ter Haar, Phys. Rev. 127, 88 (1962).

19 S. V. Tyablikov, Methods in the Quantum Theory of Magnetism (Plenum Press, New York, 1967).

20 W. Nolting and A. Ramakanth, Quantum Theory of Magnetism (Springer, 2009).

21 G. Bouzerar, T. Ziman, and J. Kudrnovský, Europhys. Lett. 69, 812 (2005).

22 G. Bouzerar, T. Ziman, and J. Kudrnovský, Phys. Rev. B 72, 125207 (2005).

23 K. Sato, L. Bergqvist, J. Kudrnovský, P. H. Dederichs, O. Eriksson, I. Turek, B. Sanyal, G. Bouzerar, H. KatayamaYoshida, V. A. Dinh, T. Fukushima, H. Kizaki, and R. Zeller, Rev. Mod. Phys. 82, 1633 (2010).

24 P. Bruno, Phys. Rev. Lett. 87, 137203 (2001).

25 N. D. Mermin and H. Wagner, Phys. Rev. Lett. 17, 1133 (1966).

26 D. Loss, F. L. Pedrocchi, and A. J. Leggett, Phys. Rev. Lett. 107, 107201 (2011).

27 R. Orbach and K. W. Yu, J. Appl. Phys. 61, 3689 (1987).

28 Y. J. Uemura and R. J. Birgeneau, Phys. Rev. Lett. 57, 1947 (1986).

29 Y. J. Uemura and R. J. Birgeneau, Phys. Rev. B 36, 7024 (1987).

30 A. Aharony, S. Alexander, O. Entin-Wohlman, and R. Orbach, Phys. Rev. B 31, 2565(R) (1985); Phys. Rev. Lett. 58, 132 (1987).

31 E. R. Mucciolo, A. H. Castro Neto, and C. Chamon, Phys. Rev. B 69, 214424 (2004).

${ }^{32}$ G. Bouzerar, Europhys. Lett. 79, 57007 (2007).

33 Y. Motome and N. Furukawa, J. Phys. Soc. Jpn. 72, 472 (2002).

34 Y. Motome and N. Furukawa, Phys. Rev. B 71, 014446 (2005). 